\title{
Siacci's Theorem for Frenet Curves in Minkowski 3-Space
}

\author{
Kahraman Esen Özen*
}

\begin{abstract}
For motion of a material point along a space curve, due to Siacci [1], a resolution of the acceleration vector is well known. In this resolution, the acceleration vector is stated as the sum of two special oblique components in the osculating plane to the curve. In this paper, we have studied the Siacci's theorem for non-relativistic particles moving along the Frenet curves at very low speeds relative to the speed of light in Minkowski 3-space. Moreover, an illustrative example is given to show how the aforesaid theorem works. This theorem is a new contribution to the field and it may be useful for some specific applications in theoretical, mathematical and computational physics.
\end{abstract}

Keywords: Kinematics of a particle; Minkowski 3-space; Plane and space curves; Siacci.

AMS Subject Classification (2020): Primary: 70B05; Secondary: 51B20; 14H50; $70 B 99$.

${ }^{*}$ Corresponding author

\section{Introduction}

The Minkowski 3-dimensional space $E_{1}^{3}$ is the Euclidean space $E^{3}$ equipped with Lorentzian inner product

$$
\langle\mathbf{X}, \mathbf{Y}\rangle_{L}=x_{1} y_{1}+x_{2} y_{2}-x_{3} y_{3}
$$

where $\mathbf{X}=\left(x_{1}, x_{2}, x_{3}\right), \mathbf{Y}=\left(y_{1}, y_{2}, y_{3}\right) \in E^{3}$. A vector $\mathbf{X} \in E_{1}^{3}$ belongs to the one of three categories which is called its causal character; spacelike, timelike and lightlike (null). The vector $\mathbf{X} \in E_{1}^{3}$ is spacelike, timelike and lightlike if $\langle\mathbf{X}, \mathbf{X}\rangle_{L}>0$ or $\mathbf{X}=0,\langle\mathbf{X}, \mathbf{X}\rangle_{L}<0$ and $\langle\mathbf{X}, \mathbf{X}\rangle_{L}=0(\mathbf{X} \neq 0)$, respectively. For a vector $\mathbf{Z}$ in $E_{1}^{3}$, the norm of $\mathbf{Z}$ is defined by $\|\mathbf{Z}\|_{L}=\sqrt{\left|\langle\mathbf{Z}, \mathbf{Z}\rangle_{L}\right|}$ and also, if $\|\mathbf{Z}\|_{L}=1, \mathbf{Z}$ is said to be a unit vector. In Minkowski 3 -space $E_{1}^{3}$, the Lorentzian vector product $\mathbf{X} \wedge{ }_{L} \mathbf{Y}$ of the vectors $\mathbf{X}=\left(x_{1}, x_{2}, x_{3}\right)$ and $\mathbf{Y}=\left(y_{1}, y_{2}, y_{3}\right)$ is defined by

$$
\mathbf{X} \wedge_{L} \mathbf{Y}=\left|\begin{array}{ccc}
e_{1} & e_{2} & -e_{3} \\
x_{1} & x_{2} & x_{3} \\
y_{1} & y_{2} & y_{3}
\end{array}\right|=\left(x_{2} y_{3}-x_{3} y_{2}, x_{3} y_{1}-x_{1} y_{3}, x_{2} y_{1}-x_{1} y_{2}\right)
$$

An arbitrary regular curve $\alpha=\alpha(t): I \subset \mathbb{R} \rightarrow E_{1}^{3}$ is said to be a spacelike curve (resp. timelike curve, lightlike curve) if all of its velocity vectors $\alpha^{\prime}(t)$ are spacelike (resp. timelike, lightlike) for each $t \in I$. If $\alpha$ is spacelike or timelike, it is called as a non-lightlike curve. In this case, $\alpha$ can be reparameterized by the arc-length $s=s(t)$ such that $\left\|\alpha^{\prime}(s)\right\|_{L}=1$. After this reparametrization $\alpha$ is called as a unit speed curve. For this unit speed curve, we can define a Frenet frame $\{\mathbf{T}(s), \mathbf{N}(s), \mathbf{B}(s)\}$ associated for each point $s$ where $\mathbf{T}(s), \mathbf{N}(s)$ and $\mathbf{B}(s)$ are called the tangent, principal normal and binormal vector fields, respectively $[2,3]$. 
Timelike curves, spacelike curves with timelike normal vector and spacelike curves with spacelike normal vector are called Frenet curves and these curves also satisfy the equations

$$
\mathbf{T}(s)=\alpha^{\prime}(s), \quad \mathbf{N}(s)=\frac{\mathbf{T}^{\prime}(s)}{\kappa(s)}, \quad \mathbf{B}(s)=\mathbf{T}(s) \wedge{ }_{L} \mathbf{N}(s)
$$

such that the orthonormal Frenet frames of timelike curves and spacelike curves with timelike normal are positively oriented and the last orthonormal Frenet frame is negatively oriented, [4]. For these curves, $\mathbf{T}^{\prime}$ is proportional to $\mathbf{N}$ and this proportion defines the curvature function $\kappa$. Also, the third coordinate of $\mathbf{N}^{\prime}$ according to Frenet basis define the torsion $\tau$, [4]. On the other hand, the Frenet formulas can be given in a unified way as in the following:

$$
\left(\begin{array}{l}
\mathbf{T}^{\prime} \\
\mathbf{N}^{\prime} \\
\mathbf{B}^{\prime}
\end{array}\right)=\left(\begin{array}{ccc}
0 & \kappa & 0 \\
\varepsilon_{\mathbf{B}} \kappa & 0 & \tau \\
0 & \varepsilon_{\mathbf{T}} \tau & 0
\end{array}\right)\left(\begin{array}{l}
\mathbf{T} \\
\mathbf{N} \\
\mathbf{B}
\end{array}\right)
$$

where $\varepsilon_{\mathbf{B}}=\langle\mathbf{B}, \mathbf{B}\rangle_{L}= \pm 1$ and $\varepsilon_{\mathbf{T}}=\langle\mathbf{T}, \mathbf{T}\rangle_{L}= \pm 1$, [5]. Some studies which are studied in the Minkowski 3-dimensional space can be found in [6-14].

On the other hand, in kinematics, the acceleration of a particle moving along a curve in space is the time derivative of its velocity. In most applications, the acceleration vector is expressed as the sum of its normal and tangential components, which are orthogonal to each other. Siacci's theorem, formulated by the Italian mathematician Francesco Siacci (1839-1907), is the kinematical decomposition of the acceleration vector into its radial and tangential components which are not generally orthogonal to each other. Firstly, F. Siacci obtained this resolution of the acceleration vector for planar motions of a particle in [15], also succeeded to obtain a smilar resolution for spatial motions of a particle in [1]. In the spatial case, the two components of the acceleration vector lie in the instantaneous osculating plane to the path of the particle. One lies along the tangent to the path, while the other one is directed from the particle towards the foot of the perpendicular that is from an arbitrary fixed origin to the instantaneous osculating plane to the path [16]. In the literature, there have been numerous studies about the Siacci's theorem. After F. Siacci, the first study about Siacci's theorem was presented by E. T. Whittaker [17]. In this study, a geometrical proof of Siacci's theorem in the plane was given. After that, a study which has a more modern proof (but the concomitant diagram was misdrawn) was put forward by N. Grossman in [18]. Recently, J. Casey has studied about the Siacci's theorem in [16]. This study has a proof of Siacci's theorem in the space which is based on the Serret-Frenet formulas. Also, for the curves in Finsler manifold $\mathbf{F}^{3}$, Siacci's theorem has been studied by Z. Küçükarslan et al. [19]. Then, K. E. Özen et al. [20] have studied the Siacci's theorem for the curves on regular surfaces in $E^{3}$ by considering the Darboux frame of a regular surface curve. Lastly, K. E. Özen et al. [21] have discussed the Siacci's theorem for the space curves which are equipped with the modified orthogonal frame.

This article is organized as follows. In Section 1 and Section 2, we have reviewed Minkowski 3-space and its some important properties needed to understand the ensuing section. In Section 3, we have studied the Siacci's theorem for a non-relativistic particle moving along the Frenet curves at very low speeds relative to the speed of light in Minkowski 3-space by inspiring the statement and proof of Siacci's theorem given in [16]. Furthermore, in this section, we have obtained the components of the acceleration for motion along a Frenet planar curve in Minkowski 3-space as a corollary and we have given an example for an application of the Siacci's theorem for Frenet curves in Minkowski 3-space.

\section{Preliminaries}

Let unit speed Frenet curves $\alpha_{i}, i=1,2,3$ be a spacelike curve with a timelike binormal, a spacelike curve with a timelike principal normal and a timelike curve, respectively and let $\mathbf{T}_{i}(s), \mathbf{N}_{i}(s), \mathbf{B}_{i}(s)$ and $\kappa_{i}(s), \tau_{i}(s)$ show the Frenet bases and the Frenet curvatures of $\alpha_{i}$. By means of (1.1), we can write the following equation for the Frenet curves $\alpha_{i}, i=1,2,3$ :

$$
\mathbf{T}_{i}(s)=\alpha_{i}{ }^{\prime}(s), \quad \mathbf{N}_{i}(s)=\frac{\mathbf{T}_{i}{ }^{\prime}(s)}{\kappa_{i}(s)}, \quad \mathbf{B}_{i}(s)=\mathbf{T}_{i}(s) \wedge_{L} \mathbf{N}_{i}(s)
$$

where the Frenet frames $\left\{\mathbf{T}_{2}, \mathbf{N}_{2}, \mathbf{B}_{2}\right\}$ and $\left\{\mathbf{T}_{3}, \mathbf{N}_{3}, \mathbf{B}_{3}\right\}$ are positively oriented and the Frenet frame $\left\{\mathbf{T}_{1}, \mathbf{N}_{1}, \mathbf{B}_{1}\right\}$ is negatively oriented. On the other hand, from (1.2), we can write the following equation for the Frenet curves $\alpha_{i}, i=1,2,3$ :

$$
\left(\begin{array}{l}
\mathbf{T}_{i}{ }^{\prime}(s) \\
\mathbf{N}_{i}{ }^{\prime}(s) \\
\mathbf{B}_{i}{ }^{\prime}(s)
\end{array}\right)=\left(\begin{array}{ccc}
0 & \kappa_{i}(s) & 0 \\
\varepsilon_{\mathbf{B}}^{\mathbf{i}}(s) \kappa_{i}(s) & 0 & \tau_{i}(s) \\
0 & \varepsilon_{\mathbf{T}}^{\mathbf{i}}(s) \tau_{i}(s) & 0
\end{array}\right)\left(\begin{array}{l}
\mathbf{T}_{i}(s) \\
\mathbf{N}_{i}(s) \\
\mathbf{B}_{i}(s)
\end{array}\right)
$$


where $\varepsilon_{\mathbf{T}}^{\mathbf{i}}(s)=\left\langle\mathbf{T}_{i}(s), \mathbf{T}_{i}(s)\right\rangle_{L}= \pm 1$ and $\varepsilon_{\mathbf{B}}^{\mathbf{i}}(s)=\left\langle\mathbf{B}_{i}(s), \mathbf{B}_{i}(s)\right\rangle_{L}= \pm 1$.

Assume that non-relativistic particles $P_{1}, P_{2}$ and $P_{3}$ with non-zero masses move asynchronously along the Frenet curves $\alpha_{1}, \alpha_{2}$ and $\alpha_{3}$ in Minkowski 3-space at very low speeds relative to the speed of light, respectively. Choose arbitrary fixed origins $O_{i}, i=1,2,3$ in the space for these three cases. Let $\mathbf{x}_{i}$ be the position vector of $P_{i}$ at time $t_{i}$, and let $s_{i}$ be the arc length of $\alpha_{i}$ described at time $t_{i}$. Then, the unit tangent vector for the curve $\alpha_{i}$ can be found as

$$
\mathbf{T}_{i}=\mathbf{x}_{i}{ }^{\prime}=\frac{d \mathbf{x}_{i}}{d s_{i}}
$$

With the aid of (2.2) and (2.3), the velocity vector $\mathbf{v}_{i}$ and the acceleration vector $\mathbf{a}_{i}$ of $P_{i}$ at time $t_{i}$ are given by

$$
\mathbf{v}_{i}=\dot{\mathbf{x}}_{i}=v_{i} \mathbf{T}_{i}, \quad \mathbf{a}_{i}=\dot{\mathbf{v}}_{i}=\dot{v}_{i} \mathbf{T}_{i}+\kappa_{i} v_{i}^{2} \mathbf{N}_{i}
$$

where $\frac{d}{d t_{i}}$ is denoted by the superposed dot and $\frac{d s_{i}}{d t_{i}}$ is symbolized by $v_{i}$. Since $\mathbf{v}_{i}$ can be expressed as a function of $s_{i}$, the acceleration of $P_{i}$ can be written as

$$
\mathbf{a}_{i}=v_{i} \frac{d v_{i}}{d s_{i}} \mathbf{T}_{i}+\kappa_{i} v_{i}^{2} \mathbf{N}_{i}
$$

Thus, the acceleration vector of $P_{i}$ always lies in the osculating plane $V_{i}$ to $\alpha_{i}$ at $P_{i}$. (Here, we follow the steps in [16] to get the acceleration vector.)

Let us denote the vector which is perpendicular in the Lorentzian sense at $P_{i}$ to the position vector $\mathbf{x}_{i}$ and the linear momentum vector $m_{i} \mathbf{v}_{i}$ by $\mathbf{H}_{i}$. It means that the equality

$$
\mathbf{H}_{i}=\mathbf{x}_{i} \wedge_{L} m_{i} \mathbf{v}_{i}=\mathbf{x}_{i} \wedge_{L} m_{i} v_{i} \mathbf{T}_{i}
$$

is satisfied.

\section{Siacci's theorem for Frenet curves}

Let the position vector of $P_{i}$ be resolved on the Serret-Frenet bases in Minkowski 3-space as follows:

$$
\mathbf{x}_{i}=q_{i} \mathbf{T}_{i}-p_{i} \mathbf{N}_{i}+b_{i} \mathbf{B}_{i} .
$$

In that case,

$$
\mathrm{q}_{i}=\varepsilon_{\mathbf{T}}^{\mathbf{i}}\left\langle\mathbf{x}_{i}, \mathbf{T}_{i}\right\rangle_{L}, \quad \mathrm{p}_{i}=-\varepsilon_{\mathbf{N}}^{\mathbf{i}}\left\langle\mathbf{x}_{i}, \mathbf{N}_{i}\right\rangle_{L}, \quad b_{i}=\varepsilon_{\mathbf{B}}^{\mathbf{i}}\left\langle\mathbf{x}_{i}, \mathbf{B}_{i}\right\rangle_{L}
$$

where $\varepsilon_{\mathbf{T}}^{\mathbf{i}}=\left\langle\mathbf{T}_{i}, \mathbf{T}_{i}\right\rangle_{L^{\prime}} \varepsilon_{\mathbf{N}}^{\mathbf{i}}=\left\langle\mathbf{N}_{i}, \mathbf{N}_{i}\right\rangle_{L}$ and $\varepsilon_{\mathbf{B}}^{\mathbf{i}}=\left\langle\mathbf{B}_{i}, \mathbf{B}_{i}\right\rangle_{L}$

Denote by $\mathbf{r}_{i}$ a vector in the osculating plane $V_{i}$ as in the following equation

$$
\mathbf{r}_{i}=-p_{i} \mathbf{N}_{i}+q_{i} \mathbf{T}_{i}
$$

Then, we get

$$
\left\langle\mathbf{r}_{i}, \mathbf{r}_{i}\right\rangle_{L}=\varepsilon_{\mathbf{N}}^{\mathbf{i}} p_{i}{ }^{2}+\varepsilon_{\mathbf{T}}^{\mathbf{i}} q_{i}{ }^{2} .
$$

By differentiating the equation (3.1) with respect to the arc length parameter $s_{i}$ and taking into consideration (2.2), we get

$$
\mathbf{T}_{i}=\left(q_{i}{ }^{\prime}-\varepsilon_{\mathbf{B}}^{\mathbf{i}} \kappa_{i} p_{i}\right) \mathbf{T}_{i}+\left(\kappa_{i} \mathrm{q}_{i}-\mathrm{p}_{i}{ }^{\prime}+\varepsilon_{\mathbf{T}}^{\mathbf{i}} \tau_{i} b_{i}\right) \mathbf{N}_{i}+\left(-\tau_{i} \mathrm{p}_{i}+\mathrm{b}_{i}{ }^{\prime}\right) \mathbf{B}_{i} .
$$

In that case, the equations

$$
q_{i}{ }^{\prime}=1+\varepsilon_{\mathbf{B}}^{\mathbf{i}} \kappa_{i} p_{i}, \quad p_{i}{ }^{\prime}=\kappa_{i} \mathrm{q}_{i}+\varepsilon_{\mathbf{T}}^{\mathbf{i}} \tau_{i} \mathrm{~b}_{i}, \quad \mathrm{~b}_{i}{ }^{\prime}=\tau_{i} \mathrm{p}_{i}
$$


hold since the Frenet bases are linearly independent. If we differentiate (3.4) and use the formulas (3.6) by considering the values of $\varepsilon_{\mathbf{T}}^{\mathbf{i}}, \varepsilon_{\mathbf{N}}^{\mathbf{i}}$ and $\varepsilon_{\mathbf{B}}^{\mathbf{i}}$, we have

$$
\left\langle\mathbf{r}_{i}, \mathbf{r}_{i}{ }^{\prime}\right\rangle_{L}=\varepsilon_{\mathbf{T}}^{\mathbf{i}} q_{i}-\varepsilon_{\mathbf{B}}^{\mathbf{i}} \tau_{i} \mathrm{p}_{i} \mathrm{~b}_{i}, \quad\left\langle\mathbf{r}_{i}, \mathbf{r}_{i}{ }^{\prime}\right\rangle_{L}=\varepsilon_{\mathbf{T}}^{\mathbf{i}} q_{i}-\varepsilon_{\mathbf{B}}^{\mathbf{i}} \mathrm{b}_{i} \mathrm{~b}_{i}{ }^{\prime} .
$$

If the vector $\mathbf{H}_{i}$, given by (2.6), is calculated by considering the position vector $\mathbf{x}_{i}$ indicated in (3.1), the vector $\mathbf{H}_{i}$ takes the form

$$
\mathbf{H}_{i}=m_{i} v_{i} \mathrm{~b}_{i} \mathbf{N}_{i}+m_{i} v_{i} \mathrm{p}_{i} \mathbf{B}_{i} .
$$

Let us use the notations as follows:

$$
h_{i}=\mathrm{p}_{i} v_{i}, \quad w_{i}=\mathrm{b}_{i} v_{i} .
$$

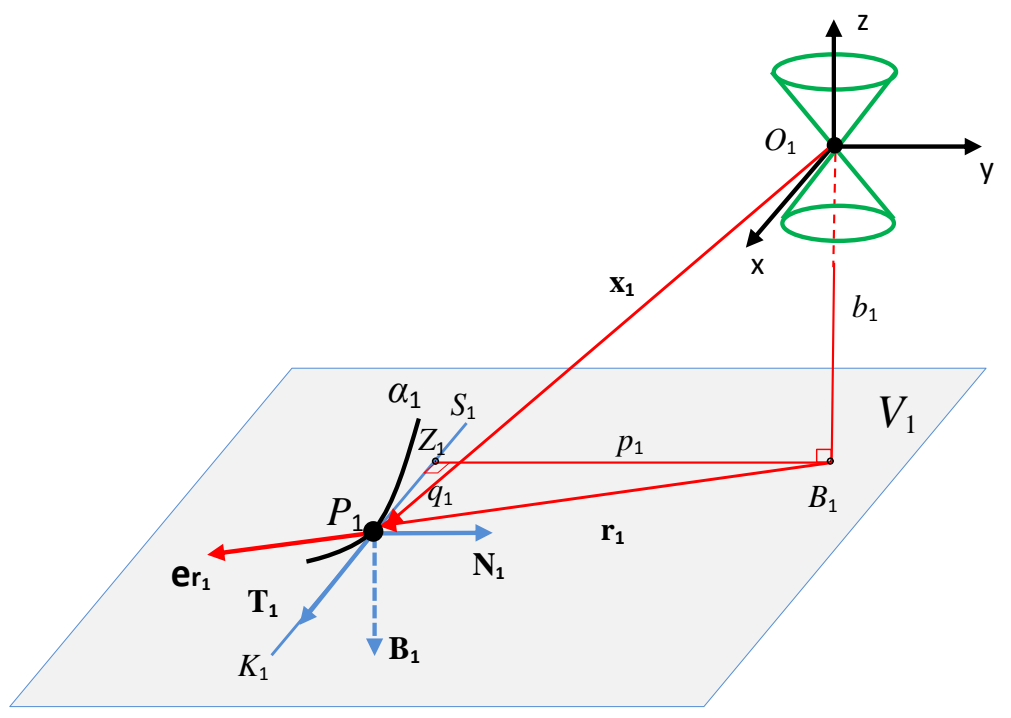

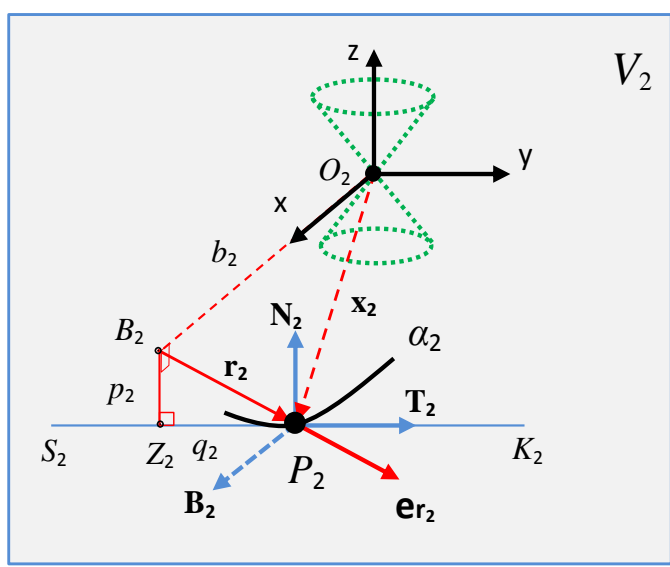

Fig. 1-B
Fig. 1-A

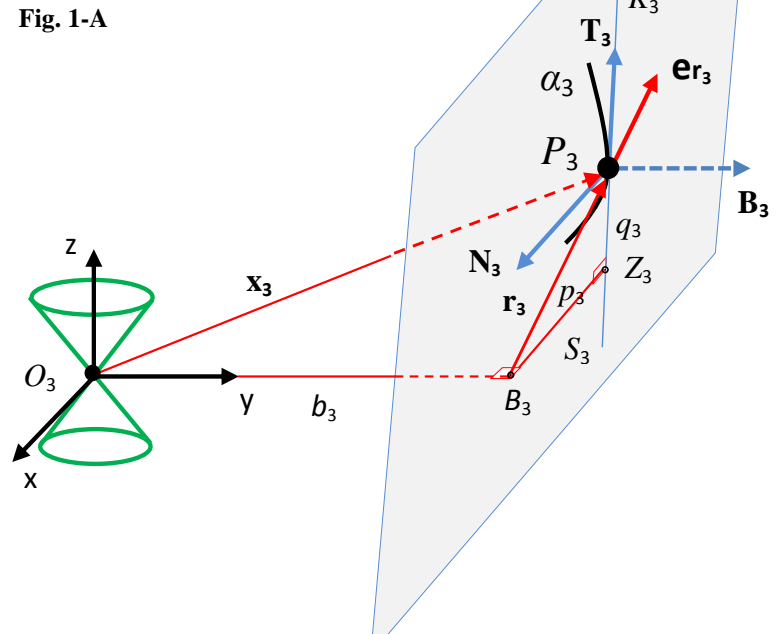

Fig. 1-C

Figure 1. The particle $P_{i}$ moves along the curve $\alpha_{i}$ in Minkowski 3-space. The osculating plane at $P_{i}$ is $V_{i}$ and the tangent line to $\alpha_{i}$ at $P_{i}$ is $S_{i} P_{i} K_{i} . O_{i} B_{i}$ is the perpendicular that is orthogonal in the Lorentzian sense from origin $O_{i}$ to the osculating plane $V_{i} . \mathbf{r}_{i}$ is the position vector of $P_{i}$ relative to $B_{i}$, and $B_{i} Z_{i}$ is perpendicular that is orthogonal in the Lorentzian sense from $B_{i}$ to the tangent line. $-\mathrm{p}_{i} \mathbf{N}_{i}$ is the position vector of $Z_{i}$ relative to $B_{i}$, and $\mathrm{q}_{i} \mathbf{T}_{i}$ is the position vector of $P_{i}$ relative to $Z_{i}$. 
We need to warn the readers about some cases. Figure 1 given above is a representative figure and however the perpendiculars in this figure seem to be orthogonal in the Euclidean sense, they represent the perpendiculars that are orthogonal in the Lorentzian sense. We prefer this to ensure the visual harmony of the figure.

According to Siacci's theorem, the acceleration vector of a particle $P$ of mass $m(>0)$ which travels along a curve $C$ in Euclidean 3-space can be written by the sum of its tangential direction and the radial direction in the osculating plane, [16]. We aim the same for the particles $P_{i}, i=1,2,3$ in Minkowski 3-space. For this purpose, firstly let us express the vector $\mathbf{N}_{i}$ in terms of $\mathbf{r}_{i}$ and $\mathbf{T}_{i}$ by using the equation (3.3). But this is possible if and only if $\mathrm{p}_{i} \neq 0$. By making an assumption "the binormal component of the vector $\mathbf{H}_{i}$ in (3.8) never vanishes", it can be easily said that $\mathrm{p}_{i} \neq 0$. Thus, from (3.3) we get

$$
\mathbf{N}_{i}=\frac{1}{\mathrm{p}_{i}}\left(-\mathbf{r}_{i}+\mathrm{q}_{i} \mathbf{T}_{i}\right) .
$$

Substituting this last equation in (2.5) we get

$$
\mathbf{a}_{i}=-\frac{\kappa_{i} v_{i}^{2}}{\mathrm{p}_{i}} \mathbf{r}_{i}+\left(v_{i} \frac{d v_{i}}{d s_{i}}+\frac{\kappa_{i} v_{i}^{2} \mathrm{q}_{i}}{\mathrm{p}_{i}}\right) \mathbf{T}_{i} .
$$

As it is seen from the equation (3.4), when $\mathrm{p}_{i}=\mathrm{q}_{i},\left\langle\mathbf{r}_{i}, \mathbf{r}_{i}\right\rangle_{L}=0$ for the curves $\alpha_{i}, i=2,3$. In this case, the unit vectors in direction of $\mathbf{r}_{i}, i=2,3$ can not be defined. By making an assumption that the vectors $\mathbf{r}_{i}, i=2,3$ in the radial directions do not lie on the light-cone, we can avert this. After this assumption, we have $\mathbf{r}_{i}=\left\|\mathbf{r}_{i}\right\|_{L}=\sqrt{\left|\left\langle\mathbf{r}_{i}, \mathbf{r}_{i}\right\rangle_{L}\right|} \neq 0, i=2,3$. On the other hand, from the equation (3.4), it is clear that $\mathbf{r}_{i}=\left\|\mathbf{r}_{i}\right\|_{L}=\sqrt{\left|\left\langle\mathbf{r}_{i}, \mathbf{r}_{i}\right\rangle_{L}\right|} \neq 0$ for $i=1$. Hence, we can define the unit vectors $\mathbf{e}_{\mathbf{r}_{\mathbf{i}}}, i=1,2,3$ in directions of $\mathbf{r}_{i}, i=1,2,3$ by

$$
\mathbf{e}_{\mathbf{r}_{i}}=\frac{1}{\mathbf{r}_{i}} \mathbf{r}_{i}
$$

If we substitute the equation (3.11) in (3.10), we have the fundamental form of Siacci's resolution of the acceleration vector in Minkowski 3-space as

$$
\mathbf{a}_{i}=-\frac{\kappa_{i} v_{i}{ }^{2} \mathrm{r}_{i}}{\mathrm{p}_{i}} \mathbf{e}_{\mathbf{r}_{i}}+\left(v_{i} \frac{d v_{i}}{d s_{i}}+\frac{\kappa_{i} v_{i}^{2} \mathrm{q}_{i}}{\mathrm{p}_{i}}\right) \mathbf{T}_{i}=\mathrm{S}_{\mathbf{r}_{i}} \mathbf{e}_{\mathbf{r}_{i}}+\mathrm{S}_{\mathbf{T}_{i}} \mathbf{T}_{i} .
$$

Here, $S_{\mathbf{r}_{i}}$ and $S_{\mathbf{T}_{i}}$ are called the radial and tangential Siacci components of the acceleration, respectively. Using the equation (3.9) with $\mathrm{p}_{i} \neq 0$, we get the radial Siacci component as follows:

$$
S_{\mathbf{r}_{i}}=-\frac{\kappa_{i} \mathrm{r}_{i} h_{i}{ }^{2}}{\mathrm{p}_{i}{ }^{3}} .
$$

Now, let us show that the tangential Siacci component can be put into three different forms. At first, if we consider the equations (3.6) and (3.9), we obtain

$$
S_{\mathbf{T}_{i}}=\frac{\left(v_{i}{ }^{2}\right)^{\prime}}{2}+\frac{v_{i}{ }^{2} \kappa_{i} \mathrm{q}_{i}}{\mathrm{p}_{i}}=\frac{\left(v_{i}{ }^{2}\right)^{\prime}}{2}+\frac{v_{i}{ }^{2} \mathrm{p}_{i}{ }^{\prime}}{\mathrm{p}_{i}}-\varepsilon_{\mathbf{T}}^{\mathbf{i}} \frac{v_{i}{ }^{2} \tau_{i} \mathrm{~b}_{i}}{\mathrm{p}_{i}}=\frac{\left(\mathrm{h}_{i}{ }^{2}\right)^{\prime}}{2 \mathrm{p}_{i}{ }^{2}}-\varepsilon_{\mathbf{T}}^{\mathbf{i}} \frac{\tau_{i} \mathrm{~b}_{i} \mathrm{~h}_{i}{ }^{2}}{\mathrm{p}_{i}{ }^{3}}=\frac{1}{2 \mathrm{p}_{i}{ }^{2}}\left\{\left(\mathrm{~h}_{i}{ }^{2}\right)^{\prime}-\varepsilon_{\mathbf{T}}{ }^{\mathbf{i}} \frac{\mathrm{h}_{i}{ }^{2}}{\mathrm{p}_{i}{ }^{2}}\left(\mathrm{~b}_{i}{ }^{2}\right)^{\prime}\right\}
$$

In a similar way, using the first expression in (3.7), we get

$$
S_{\mathbf{T}_{i}}=\frac{1}{2}\left(v_{i}^{2}\right)^{\prime}+\kappa_{i} v_{i}^{2}\left\{\varepsilon_{\mathbf{T}}^{\mathbf{i}} \frac{\left\langle\mathbf{r}_{i}, \mathbf{r}_{i}\right\rangle_{L}^{\prime}}{2 \mathrm{p}_{i}}-\varepsilon_{\mathbf{N}}^{\mathbf{i}} \tau_{i} \mathrm{~b}_{i}\right\} .
$$

The following two equations appear depending on the causal character of the vector $\mathbf{r}_{i}$. That is, if the vector $\mathbf{r}_{i}$ is spacelike or timelike, the equations (3.16) and (3.17) hold respectively:

$$
\begin{aligned}
& S_{\mathbf{T}_{i}}=\frac{1}{2}\left(v_{i}^{2}\right)^{\prime}+\kappa_{i} v_{i}^{2}\left\{\varepsilon_{\mathbf{T}}^{\mathbf{i}} \frac{\left(\mathrm{r}_{i}^{2}\right)^{\prime}}{2 \mathrm{p}_{i}}-\varepsilon_{\mathbf{N}}^{\mathbf{i}} \tau_{i} \mathrm{~b}_{i}\right\}, \\
& S_{\mathbf{T}_{i}}=\frac{1}{2}\left(v_{i}^{2}\right)^{\prime}-\kappa_{i} v_{i}{ }^{2}\left\{\varepsilon_{\mathbf{T}}^{\mathbf{i}} \frac{\left(\mathrm{r}_{i}^{2}\right)^{\prime}}{2 \mathrm{p}_{i}}+\varepsilon_{\mathbf{N}}^{\mathbf{i}} \tau_{i} \mathrm{~b}_{i}\right\} .
\end{aligned}
$$


Finally, if we take into consideration the second expression in (3.7), we obtain

$$
S_{\mathbf{T}_{i}}=\frac{1}{2}\left(v_{i}^{2}\right)^{\prime}+\frac{\kappa_{i} v_{i}^{2}}{2 \mathrm{p}_{i}}\left[\varepsilon_{\mathbf{T}}^{\mathbf{i}}\left\langle\mathbf{r}_{i}, \mathbf{r}_{i}\right\rangle_{L}-\varepsilon_{\mathbf{N}}^{\mathbf{i}}{b_{i}}^{2}\right]^{\prime}
$$

Likewise, if the vector $\mathbf{r}_{i}$ is spacelike or timelike, the equations (3.19) and (3.20) hold respectively:

$$
\begin{aligned}
& S_{\mathbf{T}_{i}}=\frac{1}{2}\left(v_{i}{ }^{2}\right)^{\prime}+\frac{\kappa_{i} v_{i}{ }^{2}}{2 \mathrm{p}_{i}}\left[\varepsilon_{\mathbf{T}^{\mathbf{i}}}^{\mathbf{i}}{ }^{2}-\varepsilon_{\mathbf{N}}^{\mathbf{i}} \mathrm{b}_{i}{ }^{2}\right]^{\prime}, \\
& S_{\mathbf{T}_{i}}=\frac{1}{2}\left(v_{i}{ }^{2}\right)^{\prime}-\frac{\kappa_{i} v_{i}{ }^{2}}{2 \mathrm{p}_{i}}\left[\varepsilon_{\mathbf{T}^{i}}^{\mathbf{i}}{ }^{2}+\varepsilon_{\mathbf{N}}^{\mathbf{i}} \mathrm{b}_{i}{ }^{2}\right]^{\prime} .
\end{aligned}
$$

If we take into consideration above derivation, we can express the following theorem and corollary for Frenet curves in Minkowski 3-space.

Theorem 3.1. (Siacci's theorem for Frenet curves) Let non-relativistic particles $P_{1}, P_{2}$ and $P_{3}$ move along a spacelike curve $\alpha_{1}$ with a timelike binormal, a spacelike curve $\alpha_{2}$ with a timelike principal normal and a timelike curve $\alpha_{3}$ at very low speeds relative to the speed of light in Minkowski 3-space, respectively. Also, let arbitrary fixed origins $O_{i}, i=1,2,3$ be chosen in the space for these three motions. Assume that the binormal component of the vector $\mathbf{H}_{i}(i=1,2,3)$, given by (3.8), never vanishes. Denote by $B_{i}(i=1,2,3)$ the foot of the perpendicular that is orthogonal in the Lorentzian sense from $O_{i}(i=1,2,3)$ to the osculating plane $V_{i}(i=1,2,3)$. Also, for the case of $i=2,3$, assume that the vector $\vec{B}_{i} \vec{P}_{i}$ does not lie on the light-cone. Then, the acceleration vector of $P_{i}(i=1,2,3)$ can be expressed as the sum of the Siacci components as in (3.12).

Corollary 3.1. The radial component $\mathrm{S}_{\mathbf{r}_{i}}$ can be written as in (3.13) except for the form in (3.12), while the tangential component $\mathrm{S}_{\mathbf{T}_{i}}$ can be written as (3.14), (3.15) and (3.18) except for the form in (3.12). Additionally, due to the causal character of the vector $\vec{B}_{i} P_{i}(i=1,2,3)$, the specific forms of the tangential component $(3.16),(3.17)$ and $(3.19),(3.20)$ which are obtained from (3.15) and (3.18), respectively exist.

In Minkowski 3-space, let the oriented Frenet curve $\alpha_{i}$ traced out by $P_{i}$ be restricted to a fixed plane which does not necessarily contain the origin $O_{i}$. From [4], we know that in Minkowski-3 space, a Frenet curve parameterized by the arc length is a planar curve if and only if the torsion vanishes. As a result of this, $\tau_{i}=0$ for the oriented curve $\alpha_{i}$ traced out by $P_{i}$. Thus the vector $\mathbf{B}_{i}$ is constant and orthogonal in the Lorentzian sense to the plane. If the equations (3.1), (3.3) and (3.11) are considered, the position vector of $P_{i}$ is given by

$$
\mathbf{x}_{i}=\mathbf{r}_{i} \mathbf{e}_{\mathbf{r}_{i}}+b_{i} \mathbf{B}_{i}
$$

Now, (3.12) holds in the plane of the motion. If we consider $\tau_{i}=0$, from (3.14) and (3.15) we obtain (3.22) and (3.23), respectively

$$
\begin{gathered}
S_{\mathbf{T}_{i}}=\frac{\left(\mathrm{h}_{i}{ }^{2}\right)^{\prime}}{2 \mathrm{p}_{i}{ }^{2}} \\
S_{\mathbf{T}_{i}}=\frac{1}{2}\left[\left(v_{i}{ }^{2}\right)^{\prime}+\kappa_{i} v_{i}{ }^{2} \varepsilon_{\mathbf{T}}^{\mathbf{i}} \frac{\left\langle\mathbf{r}_{i}, \mathbf{r}_{i}\right\rangle_{L}^{\prime}}{\mathrm{p}_{i}}\right] .
\end{gathered}
$$

Hence, the following corollary can be given.

Corollary 3.2. In Minkowski 3-space, let the oriented Frenet curve $\alpha_{i}$ traced out by $P_{i}$ be restricted to a fixed plane which does not necessarily contain the origin $O_{i}$. Assume that the binormal component of the vector $\mathbf{H}_{i}$ never vanishes and that $\overrightarrow{B_{i} P_{i}}$ does not lie on the light-cone. Then, Siacci's resolution (3.12) holds and the tangential Siacci component of the acceleration takes the forms (3.22) and (3.23).

It may be noted that if $\mathrm{b}_{i}=0$, the fixed plane in Corollary 3.2 obviously passes through $O_{i}$. 
Example 3.1. Suppose that a particle travels along an Euclidean helix $\alpha$ lying on a cylinder of radius $A$ at very low speed relative to the speed of light. Let the parametric equation of $\alpha$ be given by

$$
\alpha(s)=\left(A \cos \frac{s}{\sqrt{B^{2}-A^{2}}}, A \sin \frac{s}{\sqrt{B^{2}-A^{2}}}, B \frac{s}{\sqrt{B^{2}-A^{2}}}\right)
$$

where $A$ and $B$ are constants which satisfy the inequality $0<A<B$. In that case, the velocity vector is given by

$$
\alpha^{\prime}(s)=\left(-\frac{A}{\sqrt{B^{2}-A^{2}}} \sin \frac{s}{\sqrt{B^{2}-A^{2}}}, \frac{A}{\sqrt{B^{2}-A^{2}}} \cos \frac{s}{\sqrt{B^{2}-A^{2}}}, \frac{B}{\sqrt{B^{2}-A^{2}}}\right) .
$$

From the equalities $\left\langle\alpha^{\prime}(s), \alpha^{\prime}(s)\right\rangle_{L}=-1$ and $\left\|\alpha^{\prime}(s)\right\|_{L}=\sqrt{\left|\left\langle\alpha^{\prime}(s), \alpha^{\prime}(s)\right\rangle_{L}\right|}=1$, it can be said that $\alpha$ is a unit speed timelike curve and $s$ is the arc length parameter of $\alpha$. So $\alpha$ is an example of the type of the curve $\alpha_{3}$. Then, we can use the equations which we have obtained for the curve $\alpha_{3}$ in this section.

Let $\mathrm{C}=\sqrt{B^{2}-A^{2}}$. Since the particle travels along $\alpha$, we can immediately write the position vector of the particle as in the following:

$$
\mathbf{x}=\left(A \cos \frac{s}{\mathrm{C}}, A \sin \frac{s}{\mathrm{C}}, B \frac{s}{\mathrm{C}}\right) .
$$

Thus, the velocity and acceleration vectors of the particle are

$$
\mathbf{v}=\left(-\frac{A v}{\mathrm{C}} \sin \frac{s}{\mathrm{C}}, \frac{A v}{\mathrm{C}} \cos \frac{s}{\mathrm{C}}, \frac{B v}{\mathrm{C}}\right), \mathbf{a}=\left(\frac{-\mathrm{C} A v \frac{d v}{d s} \sin \frac{s}{\mathrm{C}}-A v^{2} \cos \frac{s}{\mathrm{C}}}{\mathrm{C}^{2}}, \frac{\mathrm{C} A v \frac{d v}{d s} \cos \frac{s}{\mathrm{C}}-A v^{2} \sin \frac{s}{\mathrm{C}}}{\mathrm{C}^{2}}, \frac{B v \frac{d v}{d s}}{\mathrm{C}}\right)
$$

where $v=\frac{d s}{d t}$.

The Frenet bases for $\alpha$, the curvature and torsion can be obtained as

$$
\begin{aligned}
\mathbf{T}(\mathbf{s}) & =\left(-\frac{A}{\mathrm{C}} \sin \frac{s}{\mathrm{C}}, \frac{A}{\mathrm{C}} \cos \frac{s}{\mathrm{C}}, \frac{B}{\mathrm{C}}\right), \\
\mathbf{N}(\mathbf{s}) & =\left(-\cos \frac{s}{\mathrm{C}},-\sin \frac{s}{\mathrm{C}}, 0\right), \\
\mathbf{B}(\mathbf{s}) & =\left(\frac{B}{\mathrm{C}} \sin \frac{s}{\mathrm{C}},-\frac{B}{\mathrm{C}} \cos \frac{s}{\mathrm{C}},-\frac{A}{\mathrm{C}}\right), \\
\kappa(s) & =\frac{A}{\mathrm{C}^{2}}, \\
\tau(s) & =\frac{B}{\mathrm{C}^{2}} .
\end{aligned}
$$

Due to the causal characters of the Frenet bases for timelike curve $\alpha$, the equalities

$$
\varepsilon_{\mathbf{T}(s)}=\langle\mathbf{T}(s), \mathbf{T}(s)\rangle_{L}=-1, \quad \varepsilon_{\mathbf{N}(s)}=\langle\mathbf{N}(s), \mathbf{N}(s)\rangle_{L}=1, \quad \varepsilon_{\mathbf{B}(s)}=\langle\mathbf{B}(s), \mathbf{B}(s)\rangle_{L}=1
$$

are obvious. Then, by considering (3.2), (3.24) and (3.26), we find the components of the position vector of the particle on the Serret-Frenet bases as

$$
\mathrm{q}(s)=\frac{B^{2}}{\mathrm{C}^{2}} s, \quad \mathrm{p}(s)=A, \quad \mathrm{~b}(s)=\frac{B A}{\mathrm{C}^{2}} s .
$$

If the equations (3.28) are substituted in (3.9), $h(s)$ and $w(s)$ are obtained as

$$
h(s)=A v, \quad w(s)=\frac{B A}{\mathrm{C}^{2}} v s .
$$

From (3.27) and (3.4), it can be noted that

$$
\mathrm{r}=\|\mathbf{r}\|_{L}=\sqrt{\left|\mathrm{p}^{2}-\mathrm{q}^{2}\right|}=\sqrt{\left|A^{2}-\frac{B^{4}}{\mathrm{C}^{4}} s^{2}\right|}=\sqrt{\left|\left(A+\frac{B^{2}}{\mathrm{C}^{2}} s\right)\left(A-\frac{B^{2}}{\mathrm{C}^{2}} s\right)\right|} .
$$


Considering this last equation, it can be said that; the values of the parameter which can make the vector $\mathbf{r}$ lightlike are $\pm \frac{A C^{2}}{B^{2}}$. Then, we conclude that Theorem 3.1 is applicable for all the values of the parameter except for $s= \pm \frac{A C^{2}}{B^{2}}$. If this situation is kept in mind, Siacci components

$$
S_{\mathbf{r}}=-\frac{v^{2}}{\mathrm{C}^{2}} \sqrt{\left|A^{2}-\frac{B^{4}}{\mathrm{C}^{4}} s^{2}\right|}, \quad S_{\mathbf{T}}=v \frac{d v}{d s}+\frac{v^{2} B^{2}}{\mathrm{C}^{4}} s
$$

of the particle's acceleration are easily obtained by applying Theorem 3.1.

\section{Conclusion}

Along a space curve in the 3-dimensional Euclidean space, a resolution of the acceleration vector (time derivative of velocity vector) is well known thanks to Siacci [1]. This resolution comprises two special oblique components which lie in the osculating plane of the curve. In kinematics and mechanics, to state the acceleration vector in this form is practical and has many advantages.

In this paper, by adapting Siacci's this idea to the Frenet curves in Minkowski 3-space, a similar resolution is obtained for the acceleration of a non-relativistic particle moving along a Frenet curve. This is a new contribution to the field. Just like in the case of Siacci's resolution, it may be needed for some specific applications in many areas of science.

\section{References}

[1] Siacci, F.: Moto per una linea gobba. Atti R Accad Sci. Torino. 14, 946-951 (1879).

[2] Babaarslan, M., Yaylı, Y.: On spacelike constant slope surfaces and Bertrand curves in Minkowski 3-space. Annals of the Alexandru Ioan Cuza University-Mathematics (2015). https:/ /doi.org/10.1515/aicu-2015-0009

[3] Ekici, C., Öztürk, H.: On time-like ruled surfaces in Minkowski 3-space. Universal Journal of Applied Science. 1 (2), 56-63 (2013).

[4] Lopez, R.: Differential geometry of curves and surfaces in Lorentz-Minkowski space. International Electronic Journal of Geometry. 7 (1), 44-107 (2014).

[5] Choi, J. H., Kimb, Y. H., Ali, A. T.: Some associated curves of Frenet non-lightlike curves in $E_{1}^{3}$. J. Math. Anal. Appl. 394 (2), 712-723 (2012).

[6] Altunkaya, B., Kula, L.: Characterizations of slant and spherical helices due to pseudo-Sabban frame. Fundamental Journal of Mathematics and Applications. 1 (1), 49-56 (2018).

[7] Şenyurt, S., Eren, K.: Frenet çatısına göre spacelike normalli spacelike Salkowski ĕgrisinden elde edilen Smarandache eğrileri. Erzincan Universitesi Fen Bilimleri Enstitüsü Dergisi. 13 (Özel Say1-I), 7-17 (2020).

[8] Çetin, E. Ç., Bektaş, M.: Some new characterizations of symplectic curve in 4-dimensional symplectic space. Commun. Adv. Math. Sci. 2 (4), 331-334 (2019).

[9] Şenyurt, S., Eren, K.: Spacelike asli normalli spacelike anti-Salkowski Ĕ̆risinin Frenet çatısına göre Smarandache eğrileri. Gümüşhane Üniversitesi Fen Bilimleri Enstitüsü Dergisi. 10 (1), 251-260 (2020).

[10] Samanc1, H. K.: Introduction to timelike uniform B-spline curves in Minkowski-3 space. Journal of Mathematical Sciences and Modelling. 1 (3), 206-210 (2018).

[11] Erişir, T., Kardag̃, N. C.: Spinor representations of involute evolute curves in E ${ }^{3}$. Fundam. J. Math. Appl. 2 (2), 148-155 (2019).

[12] Ersoy, S., Eren, K.: Timelike tangent developable surfaces and Bonnet surfaces. Abstract and Applied Analysis. 2016, 1-7 (2016).

[13] Tuğ, G., Özdemir, Z., Aydın, S. H., Ekmekci, F. N.: Accretive growth kinematics in Minkowski 3-space. International Journal of Geometric Methods in Modern Physics. 14 (05), 1750069 (2017). 
[14] Yıldız, Ö. G., Hizal, S., Akyig̃it, M.: Type I+ helicoidal surfaces with prescribed weighted mean or Gaussian curvature in Minkowski space with density. An. St. Univ. Ovidius Constanta. 26 (3), 99-108 (2018).

[15] Siacci, F.: Moto per una linea piana. Atti R Accad Sci. Torino. 14, 750-760 (1879).

[16] Casey, J.: Siacci's resolution of the acceleration vector for a space curve. Meccanica. 46 (2), 471-476 (2011).

[17] Whittaker, E. T.: A Treatise on the analytical dynamics of particles and rigid bodies. Cambridge University Press. New York (1944).

[18] Grossman, N.: The sheer joy of celestial mechanics. Birkhäuser. Basel (1996).

[19] Küçükarslan, Z., Y1lmaz, M. Y., Bektaş, M.: Siacci's theorem for curves in Finsler manifold $\boldsymbol{F}^{3}$. Turkish Journial of Science and Technology. 7 (2), 181-185 (2012).

[20] Özen, K. E., Tosun, M., Akyig̃it, M.: Siacci's theorem according to Darboux frame. An. St. Univ. Ovidius Constanta. 25 (3), 155-165 (2017).

[21] Özen, K. E., Güner, M., Tosun, M.: A note on the acceleration and jerk in motion along a space curve. An. St. Univ. Ovidius Constanta. 28 (1), 151-164 (2020).

\section{Affiliations}

KAHRAMAN ESEN ÖZEN

ADDRESS: Sakarya-Turkey.

E-MAIL: kahraman.ozen1@ogr.sakarya.edu.tr

ORCID ID: 0000-0002-3299-6709 